This is the accepted version of the following article: $G D$ Angel, G Haritos, A Chrysanthou \& V Voloshin, "Chord line force versus displacement for thin shallow arc pre-curved bimetallic strip", Journal of Mechanical Engineering Science, Vol. 229(1): 116-124, first published online April 29, 2014, published by SAGE Publishing. All rights reserved.

The version of record is available online at doi: http://dx.doi.org/10.1177/0954406214530873 


\title{
Chord Line Force vs Displacement for Thin Shallow Arc Pre-Curved Bimetallic Strip
}

G.D. Angel, G. Haritos, A. Chrysanthou, V.Vishoshin

School of Engineering and Technology

University of Hertfordshire

College Lane,

Hatfield,

Herts AL10 9AB

U.K.

Corresponding author:

G.D. Angel, School of Engineering and Technology, University of Hertfordshire,

College Lane, Hatfield, Herts AL10 9AB, U.K.

Email: g.d.angel@herts.ac.uk

Tel.: 00441707284586

\begin{abstract}
A formula has been derived to enable the prediction of the chord line force versus displacement for a shallow arc, thin, pre-curved bimetallic strip. A pre-curved bimetallic strip that is applied with a force in an axial orientation, that is, along its chord line, exhibits nonlinear force displacement characteristics. For thin bimetallic strips, whereby the radius of curvature is large compared to the thickness of the strip, the nonlinear relationship is parabolic in nature. The new theoretical formula introduced here, were correlated to the results of a set of force displacement tests and a good overall fit of the theory to the test data, was achieved. The formula put forward in this work enables the evaluation of large chord line displacements but is limited to the permissible stress limits of the material. This work can also be directly applied to thin shallow arc beams of a single material. The application of this work is in the field of bimetallic force displacement actuators.
\end{abstract}

Key words: pre-curved bimetallic strip, chord line force, nonlinear, formula, thin 


\section{Introduction}

Castigliano second energy theorem, according to (DAHLBERG.T 2004), enables the evaluation of small beam displacements and includes simple bending theory in its application. Simple bending theory is the limiting factor when applying Castigliano theorem and the resulting formula is only good for small order displacements. For the chord line displacement of a pre-curved beam that is part of a true arc of a circle, Castigliano theorem enables the calculation of the semi-circular arc displacement along the diameter, but even when the application of Castigliano is used for shallow arcs, it can only be used for small displacements due to the inherent limitations of the simple bending theory within its integration. This work concentrates on very shallow arcs, whereby the radius of curvature is significantly larger than the radius of curvature at full circle for the same semi-circle arc length. The formula derived here, enable the evaluation of large chord line displacements as a function of an applied force, and holds true for all thin bimetallic arcs from very shallow, to full circle arcs. Fig.1 shows a side view of a shallow arc pre-curved bimetallic strip mounted such that a degree of rotational freedom exists at both ends, but end "A" is also fixed against displacement along the chord line axis. Although shown quite thick, the thickness of the bimetallic strip is considered to be thin, and in the region of a thickness to radius ratio of 100: 1 or larger. In Fig.1, $\alpha 1$ and $\alpha 2$ are the coefficients of linear expansion for each material making up the bimetallic strip, whereby $\alpha 2$ is numerically higher than $\alpha 1$.From the geometry, chord length L can easily be obtained as a function of arc length $\boldsymbol{A}_{\boldsymbol{b}}$ and radius of curvature $\mathrm{R}$.

This work will introduce the theory and then go on to prove the effectiveness of theory against actual bimetallic test data results.

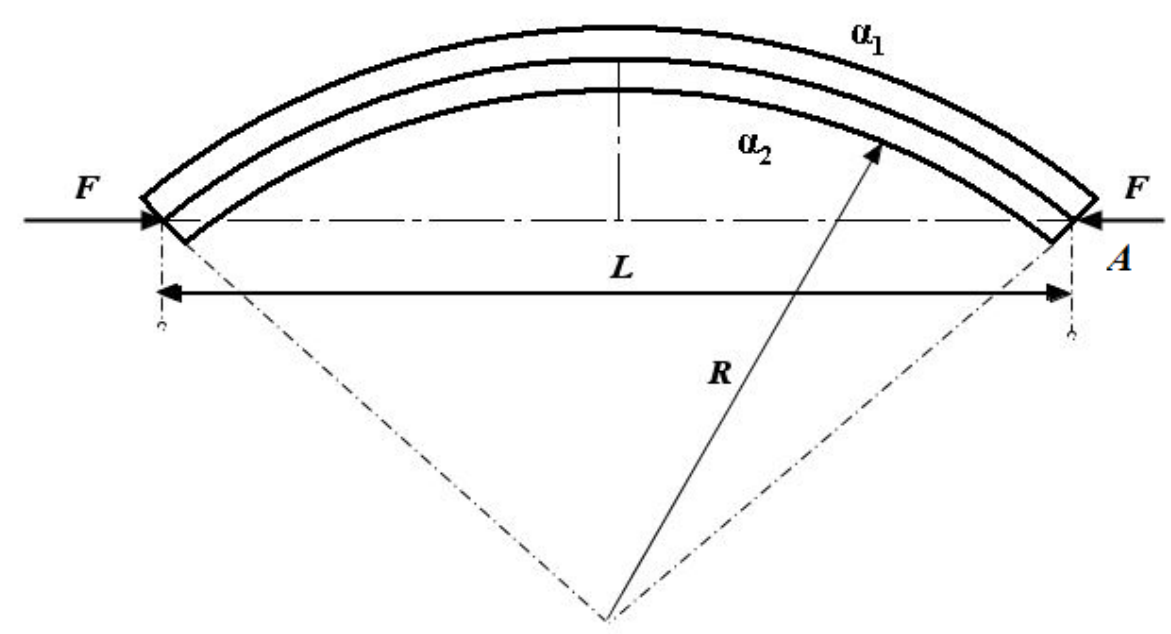

Fig.1 Pre-Curved Bimetallic Strip Material

The underlying geometry of the pre-curved bimetallic strip is shown is in Fig.2, where the three important radii of curvature are defined. It should be noted that all three arcs of Fig. 2 are the same length $\boldsymbol{A}_{\boldsymbol{b}}$. 


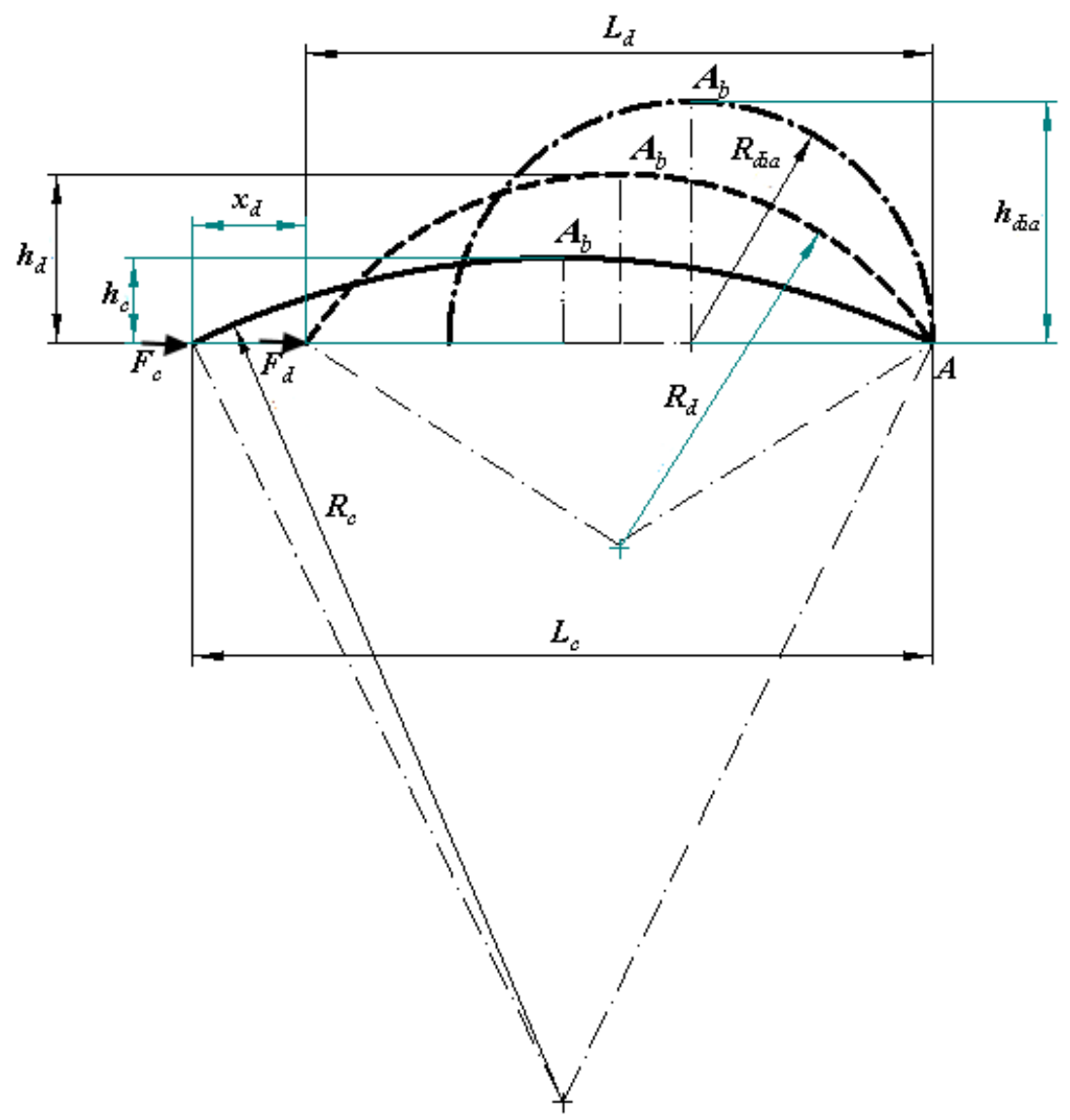

Fig. 2 Defining force displacement geometry

\section{Theory and calculations}

The following sections will introduce new methods and formulae that enable the calculation of the chord line force vs displacement for thin, shallow arc, pre-curved bimetallic strips. The derivation in the following section facilitates the calculation of the radius of curvature to displacement relationship that is crucial in evaluating the force displacement formula in later sections. Castigliano theorem enables the calculations of force vs displacement, but is limited to small displacements. This work introduces a mechanism that can cope with larger displacements and enables an accurate prediction of the forces when non-linear displacements are found.

\subsection{The non-linear $R_{d}$ vs $x_{d}$ derivation}

From Fig .1 the general chord length of any arc is generally published as

$$
L=2 R \sin \left(\frac{\theta}{2}\right)[2] \text { eqn.(1) }
$$

where: $\boldsymbol{L}$ is the chord length $(\mathrm{mm})$,

$\boldsymbol{R}$ is the radius of curvature (mm),

$\boldsymbol{A}$ is the arc length part of a true circle (mm) and

$\boldsymbol{\theta}$ is the angle subtending the portion of $\operatorname{arc} \mathbf{A}$ over distance $\boldsymbol{L}$ (radian). 
By using the established relationship: arc = radius x angle (in radians), $\boldsymbol{A}=\boldsymbol{R} \boldsymbol{\theta}$ and substituting for $\boldsymbol{\theta}=\frac{\boldsymbol{A}}{\boldsymbol{R}}$ into eqn.(1) angle $\boldsymbol{\theta}$ can be eliminated and the length of a chord $\boldsymbol{L}$ as a function of arc length $\boldsymbol{A}$ and radius of curvature $\boldsymbol{R}$, can be obtained. Denoting the lengths by suffixes b,c and d :

Thus,

$$
L_{c}=2 R_{c} \sin \left(\frac{A_{b}}{2 R_{c}}\right) \text { eqn.(2) }
$$

And thus

$$
L_{d}=2 R_{d} \sin \left(\frac{A_{b}}{2 R_{d}}\right) \text { eqn.(3) }
$$

Where suffix "b" is the bent arc length in Fig.2 in (mm)

Where suffix "c" is the initial pre-curved "cold" chord length in (mm)

Where suffix "d" is displaced chord length in $(\mathrm{mm})$ as a function of an externally applied load $\boldsymbol{F}$.

Where suffix "dia" denotes arc geometry at the diameter of the circle.

From Fig. 2, it follows that : $\quad \boldsymbol{x}_{\boldsymbol{d}}=\boldsymbol{L}_{\boldsymbol{c}}-\boldsymbol{L}_{\boldsymbol{d}}$ eqn.(4)

Where $\boldsymbol{x}_{\boldsymbol{d}}$ is the displacement from the initial position $\boldsymbol{L}_{\boldsymbol{c}}$ as a result of an applied load $\boldsymbol{F}(\mathrm{N})$.

For the evaluation of the Force vs Displacement relationship, the relationship between displaced radius of curvature $\boldsymbol{R}_{\boldsymbol{d}}$ and displacement $\boldsymbol{x}_{\boldsymbol{d}}$ first needs to be established.

From eqn.(3) and eqn.(4) and by substitution :

$$
L_{c}-x_{d}=2 R_{d} \sin \left(\frac{A_{b}}{2 R_{d}}\right) \text { eqn.(5) }
$$

But what is required is $\boldsymbol{R}_{\boldsymbol{d}}$ as function of $\boldsymbol{x}_{\boldsymbol{d}}$, which is the non-linear influence on the force displacement relationship. From eqn.(5) it can be seen that it is not possible to explicitly express $\boldsymbol{R}_{\boldsymbol{d}}$ as a function of $\boldsymbol{x}_{\boldsymbol{d}}$. The $" \sin \left(\frac{A_{b}}{2 \boldsymbol{R}_{\boldsymbol{d}}}\right)$ " term of eqn.(5) can be expanded in the Taylor series:

The general rule for a sine expansion using Taylor series is published as:

$$
\sin (x) \approx \sum_{n=0}^{\rightarrow \infty} \frac{(-1)^{n}}{(2 n+1) !} x^{(2 n+1)} \text { eqn.(6) (Abramowitz.M 1972) }
$$

And thus applying for $\boldsymbol{x}$ :

$$
\sin (x) \approx x-\frac{x^{3}}{6}+\frac{x^{5}}{120}-\frac{x^{7}}{\mathbf{5 0 4 0}} \text { eqn.(7) (Abramowitz.M 1972) }
$$

Now substituting $\frac{A_{b}}{2 R_{d}}$ into $x$ of eqn.(7) and expanding until the 4th term, this becomes:

$$
L_{c}-x_{d} \approx 2 R_{d}\left(\frac{A_{b}}{2 R_{d}}-\frac{1}{6 * 8} \frac{A_{b}^{3}}{R_{d}^{3}}+\frac{1}{120 * 32} \frac{A_{b}^{5}}{R_{d}^{5}}-\frac{1}{5040 * 128} \frac{A_{b}^{7}}{R_{d}^{7}}\right) \text { eqn.(8) }
$$

Using the substitution of $\boldsymbol{\gamma}=\frac{\mathbf{1}}{\boldsymbol{R}_{\boldsymbol{d}}^{2}}$ into eqn.(8) simplifying and rearranging becomes: 


$$
\frac{A_{b}^{6}}{\mathbf{5 0 4 0 * 6 4}} \gamma^{3}-\frac{A_{b}^{4}}{120 * 16} \gamma^{2}+\frac{A_{b}^{2}}{6 * 4} \gamma+\frac{L_{c}-x_{d}-A_{b}}{A_{b}}=0 \text { eqn.(9) }
$$

$\operatorname{Eqn}(9)$ is a cubic equation of the general form :

$$
\boldsymbol{a} \boldsymbol{\gamma}^{3}+\boldsymbol{b} \boldsymbol{\gamma}^{2}+\boldsymbol{c} \boldsymbol{\gamma}+\boldsymbol{d}=\mathbf{0}
$$

Eqn(9) can be solved by finding the discriminant and coefficients in the equation.

The discriminant for a cubic equation is generally published as :

$$
\Delta=\mathbf{1 8} \boldsymbol{a} \boldsymbol{b} \boldsymbol{c} d-\mathbf{4} \boldsymbol{b}^{3} \boldsymbol{d}+\boldsymbol{b}^{2} \boldsymbol{c}^{2}-\mathbf{4} \boldsymbol{a} \boldsymbol{c}^{2}-\mathbf{2 7} \boldsymbol{a}^{2} \boldsymbol{d}^{2} \text { eqn.(11) (Abramowitz.M 1972) }
$$

Where $\boldsymbol{a}, \boldsymbol{b}, \boldsymbol{c}, \boldsymbol{d}$ are the coefficients of eqn.(10) and are equal to:

$$
\begin{aligned}
& \boldsymbol{a}=\frac{\boldsymbol{A}_{\boldsymbol{b}}^{\mathbf{6}}}{\mathbf{5 0 4 0} * \mathbf{6 4}} \text { eqn.(12) } \\
& b=-\frac{A_{b}^{4}}{120 * 16} \quad \text { eqn.(13) } \\
& \boldsymbol{c}=\frac{A_{b}^{2}}{\mathbf{6} * \mathbf{4}} \quad \text { eqn.(14) } \\
& d=\frac{\boldsymbol{L}_{c}-x_{d}-A_{b}}{A_{b}} \text { eqn.(15) }
\end{aligned}
$$

In this particular case we have the condition that $\Delta<0$ which means that the equation has only one real root and also two complex conjugate roots. Due to physical nature of the results required, only the real root has significance in the solving of the equation.

The roots of the eqn.(9), in terms of the coefficients, can be solved by the published general formula for solving cubic equations:

Where

$$
\begin{aligned}
& \gamma_{k=-\frac{1}{3 a}\left(b+u_{k} C+\frac{\Delta_{0}}{u_{k} C}\right)} \text { eqn.(16) } \\
& \boldsymbol{c}=\sqrt[3]{\frac{\Delta_{1}+\sqrt{\Delta_{1}^{2}-4 \Delta_{0}^{3}}}{2}} \text { eqn.(17) }
\end{aligned}
$$

With

$$
\begin{aligned}
& \Delta_{0}=b^{2}-3 a c \quad \text { eqn.(18) } \\
& \Delta_{1}=2 b^{3}-9 a b c+27 a^{2} d \text { eqn.(19) }
\end{aligned}
$$

And

$$
\Delta_{1}^{2}-4 \Delta_{0}^{3}=-27 a^{2} \Delta \quad \text { eqn.(20) }
$$

Where $\Delta$ is the discriminant in shown in eqn.(11) earlier.

And the three cube roots are unity are therefore :

$$
u_{1}=1 ; \quad u_{2}=\frac{-1+i \sqrt{3}}{2} ; u_{3}=\frac{-1-i \sqrt{3}}{2} \text { eqn.(21) }
$$

With $\boldsymbol{k}=\mathbf{1}, \mathbf{2 , 3}$ substituted into eqn.(16) 
From experimental test calculations the root $\gamma_{2}$ of which $\boldsymbol{u}_{2}$ is the real root that best fits the application requirements.

Thus from the earlier substitution of $\boldsymbol{\gamma}=\frac{\mathbf{1}}{\boldsymbol{R}_{\boldsymbol{d}}^{2}}$ into eqn.(8) by simple rearrangement in terms of radius of curvature, thus

$$
\boldsymbol{R}_{\boldsymbol{d}}=\frac{\mathbf{1}}{\sqrt{\gamma_{\mathbf{2}}}} \text { eqn.(22) }
$$

The above equations were programmed into an Electronic spread sheet and the formulae were found to hold true for the whole range of values and possible combinations of parameters. Catia R19 Solid modelling software was used to test the values which accorded to the theory with a high degree of accuracy. The relationships just derived, enables the accommodation of the non-linear displacement characteristics by modifying the initial radius of curvature $\boldsymbol{R}_{\boldsymbol{c}}$ to reflect the changed radius of curvature $\boldsymbol{R}_{\boldsymbol{d}}$ as a function of displacement $\boldsymbol{x}_{\boldsymbol{d}}$.

$\boldsymbol{R}_{\boldsymbol{d}}$ is used to evaluate the force displacement equation in the next section.

Using the formulae just derived a $\boldsymbol{R}_{\boldsymbol{d}} \boldsymbol{v} \boldsymbol{s} \boldsymbol{x}_{\boldsymbol{d}}$ curve was plotted out using the following initial conditions :

$$
\begin{aligned}
& A_{b}=100 \mathrm{~mm} \text { arc length } \\
& R_{c}=\mathbf{1 0 0 m m} \text { initial radius of curvature of the arc }
\end{aligned}
$$

The results of the calculation are shown in Fig.3 which clearly show the nonlinear relationship.

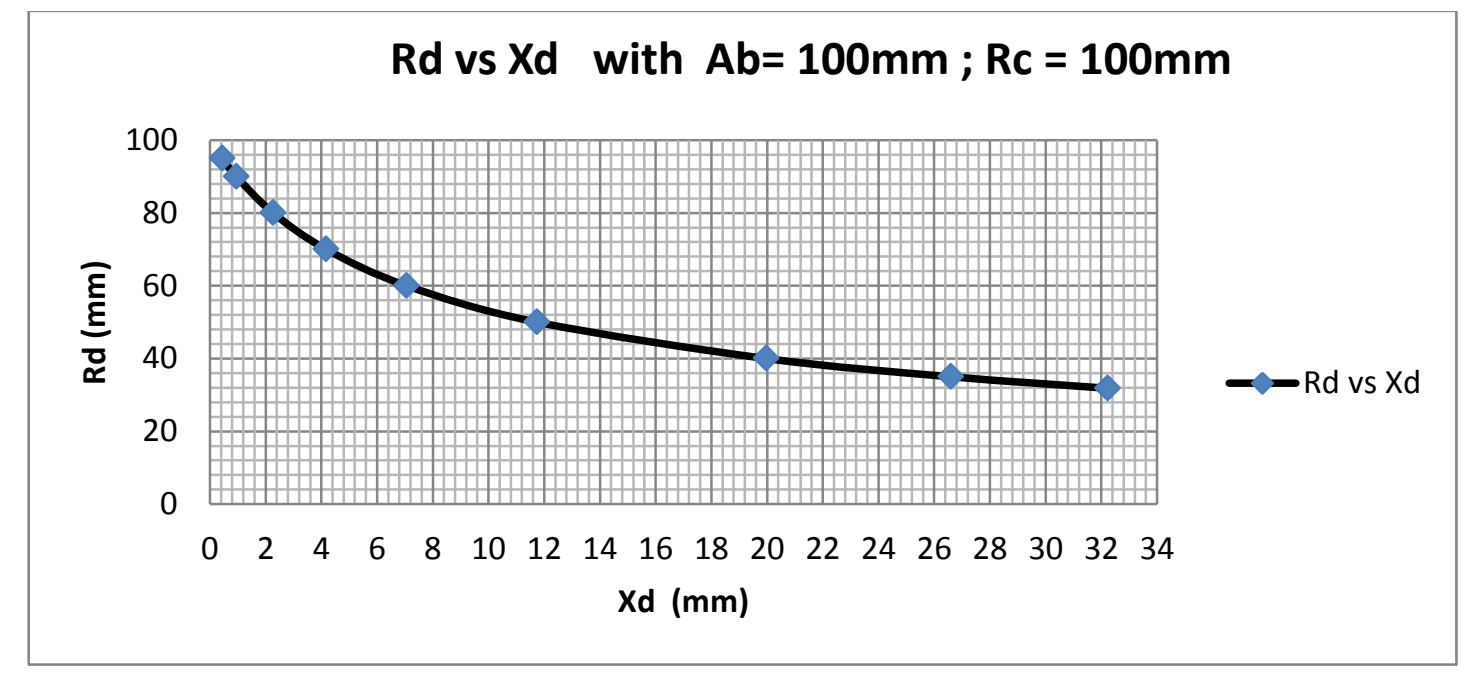

Fig. $3 R_{d} v s x_{d}$ plotted out.

\subsection{Evaluation of Force vs Displacement using $R_{d}$ as a function of $x_{d}$}

The derivation of the force displacement formula is based upon simple bending theory and the new evaluation of $\boldsymbol{R}_{\boldsymbol{d}}$ as a function of displacement $\boldsymbol{x}_{\boldsymbol{d}}$. The method introduces an imaginary force $\boldsymbol{F}_{\boldsymbol{c}}$ that is considered to be the equilibrium force required to hold the bimetallic strip at the initial radius of curvature $\boldsymbol{R}_{\boldsymbol{c}}$ when an axial load $\boldsymbol{F}_{\boldsymbol{c}}$ is applied to free end of the flat strip. The imaginary force $\boldsymbol{F}_{\boldsymbol{c}}$ 
yields an imaginary moment $\boldsymbol{M}_{\boldsymbol{c}}$ when multiplied by the distance $\boldsymbol{h}_{\boldsymbol{c}}$, see Fig.2. The imaginary moment in simple bending terms is:

$$
\boldsymbol{M}_{\boldsymbol{c}}=\frac{\boldsymbol{E} \boldsymbol{I}}{\boldsymbol{R}_{\boldsymbol{c}}} \quad \text { eqn.(23) }
$$

Likewise as result of applied real force $\boldsymbol{F}_{\boldsymbol{d}}$

$$
\boldsymbol{M}_{\boldsymbol{d}}=\frac{\boldsymbol{E} \boldsymbol{I}}{\boldsymbol{R}_{\boldsymbol{d}}} \quad \text { eqn.(24) }
$$

Where $\boldsymbol{M}_{\boldsymbol{d}} \& \boldsymbol{M}_{\boldsymbol{c}}$ are bending moments (J)

$\boldsymbol{E}$ is the Youngs Modulus for the bimetallic strip $\left(\frac{\mathrm{N}}{\mathrm{mm}^{2}}\right)$

$\boldsymbol{I}$ is the second moment of area of the cross section of the strip $\left(\mathrm{mm}^{4}\right)$

The difference between the two energy states $\boldsymbol{M}_{\boldsymbol{d}} \& \boldsymbol{M}_{\boldsymbol{c}}$ are equated to the force $\boldsymbol{F}_{\boldsymbol{d}} \mathbf{x} \boldsymbol{h}_{\boldsymbol{d}}$

Thus the displaced force is given by:

$$
\boldsymbol{F}_{\boldsymbol{d}}=\frac{\boldsymbol{M}_{\boldsymbol{d}}-\boldsymbol{M}_{\boldsymbol{c}}}{\boldsymbol{h}_{\boldsymbol{d}}}(N) \text { eqn.(25) }
$$

Where $\boldsymbol{h}_{\boldsymbol{d}}$ can easily be derived using the given geometry, thus

$$
\boldsymbol{h}_{d}=\frac{\boldsymbol{L}_{d}}{2} \tan \left(\frac{\boldsymbol{A}_{\boldsymbol{b}}}{\mathbf{4 R _ { d }}}\right)(\mathrm{mm}) \text { eqn.(26) }
$$

But from eqn. (4) previously $\boldsymbol{x}_{\boldsymbol{d}}=\boldsymbol{L}_{\boldsymbol{c}}-\boldsymbol{L}_{\boldsymbol{d}}$

Thus eqn.(25) can be written in terms of displacement $\boldsymbol{x}_{\boldsymbol{d}}$.

Hence

$$
\boldsymbol{F}_{\boldsymbol{d}}=\frac{\boldsymbol{M}_{\boldsymbol{d}}-\boldsymbol{M}_{\boldsymbol{c}}}{\frac{\left(\boldsymbol{L}_{\boldsymbol{c}}-\boldsymbol{x}_{\boldsymbol{d}}\right)}{2} \tan \left(\frac{A_{\boldsymbol{b}}}{4 \boldsymbol{R}_{\boldsymbol{d}}}\right)}(N) \text { eqn. }(27)
$$

Thus the force $\boldsymbol{F}_{\boldsymbol{d}}$ is evaluated as a function of the change in moments $\boldsymbol{M}_{\boldsymbol{d}} \& \boldsymbol{M}_{\boldsymbol{c}}$, one real, one imaginary, divided by the height $\boldsymbol{h}_{\boldsymbol{d}}$ which is a function of $\boldsymbol{R}_{\boldsymbol{d}}, \boldsymbol{x}_{\boldsymbol{d}}, \boldsymbol{L}_{\boldsymbol{c}}$, and arc length $\boldsymbol{A}_{\boldsymbol{b}}$.

This is the force vs displacement equation that overcomes the limitations of simple bending theory, and small displacements, via the introduction of $\boldsymbol{R}_{\boldsymbol{d}}$ as a function of displacement $\boldsymbol{x}_{\boldsymbol{d}}$ which accommodates the nonlinear characteristics of the thin pre-curved beam geometry.

In using eqn.(27), it is normal to know $\boldsymbol{A}_{\boldsymbol{b}}, \boldsymbol{L}_{\boldsymbol{c}}$ and $\boldsymbol{R}_{\boldsymbol{c}}$ as the initial pre-curved conditions of the bimetallic strip. By inputting a displacement $\boldsymbol{x}_{\boldsymbol{d}}$ from the initial length $\boldsymbol{L}_{\boldsymbol{c}}, \boldsymbol{F}_{\boldsymbol{d}}$ is evaluated.

In practice the amount of displacement $\boldsymbol{x}_{\boldsymbol{d}}$ of the pre-curved bimetallic strip, is limited to the permissible stresses normally defined by the materials properties of the bimetallic.

\subsection{Summary of theory}

From section 2.1 a new method of obtaining the $R_{d} v s x_{d}$ relationship was introduced. This resulted in a mechanism to evaluate the non- linearity that limits simple bending theory.

The work from 2.1 was used in the force displacement derivation in section 2.2. The theoretical equations put forward in the previous sections were programmed into an electronic worksheet and theoretical values corresponding to test samples were generated. Testing of pre-curved bimetallic strip 


\section{Test methodology}

For the experimental investigation, two independent test sets were created and tested.

The object of the tests was to verify the new formulae proposed in the earlier sections. In order to ascertain the robustness of the force deflection formulae two geometry test sets were used.

Test set 1 comprised of $\mathbf{7}$ bimetallic strips all with the same initial radius of curvature $\boldsymbol{R}_{\boldsymbol{c}} \mathbf{=} \mathbf{8 0 \mathbf { m m }}$,

Test set 2 comprised of 5 bimetallic strips of constant arc length $\boldsymbol{A}_{\boldsymbol{b}}=\mathbf{1 0 0} \mathbf{m m}$, see Table 1 .

\begin{tabular}{|l|l|l|}
\hline Test set 1 $\begin{array}{r}R_{c}=80 \mathrm{~mm} \\
\text { constant }\end{array}$ & Test set 2 $\begin{array}{c}A_{b}=100 \mathrm{~mm} \\
\text { constant }\end{array}$ \\
$A_{b}=80 \mathrm{~mm}$ & $R_{c}=50 \mathrm{~mm}$ \\
$A_{b}=100 \mathrm{~mm}$ & $R_{c}=64 \mathrm{~mm}$ \\
$A_{b}=120 \mathrm{~mm}$ & $R_{c}=70 \mathrm{~mm}$ \\
$A_{b}=140 \mathrm{~mm}$ & $R_{c}=80 \mathrm{~mm}$ \\
$A_{b}=160 \mathrm{~mm}$ & $R_{c}=84 \mathrm{~mm}$ \\
$A_{b}=180 \mathrm{~mm}$ & \\
$A_{b}=200 \mathrm{~mm}$ & \\
\hline
\end{tabular}

Table 1 Test sets $1 \& 2$

\subsection{Test setup}

For both test sets, bimetallic test samples comprising of Nickel steel and Invar 36 were made from initially straight bimetallic strip supplied by Shivalik SBC-206-1. The thickness of each metal was $0.2 \mathrm{~mm}$ and thus the total thickness of each bimetallic strip was $0.4 \mathrm{~mm}$, the width of the strips was $5 \mathrm{~mm}$. The Young's Modulus of the steel is $213 \mathrm{GN} / \mathrm{m}^{2}$ and that of Invar 36 is $145 \mathrm{GN} / \mathrm{m}^{2}$. The coefficient of linear expansion for steel is $20 \times 10^{-6} / \mathrm{K}$ and for Invar 36 is $1.85 \times 10^{-6} / \mathrm{K}$. For both test sets, all the bimetallic strips were cut back to the sizes as indicated in the two test sets, then they were gently cold formed into their radius of curvatures according to the test set sizes. After the final sizes of each test sample were checked for length and radius of curvature see Fig.5 using a set of similar formers. Each test sample was stress relieved soaked by heat treatment for 2 hours at $350^{\circ} \mathrm{C}$ according to (Kanthal 2008).

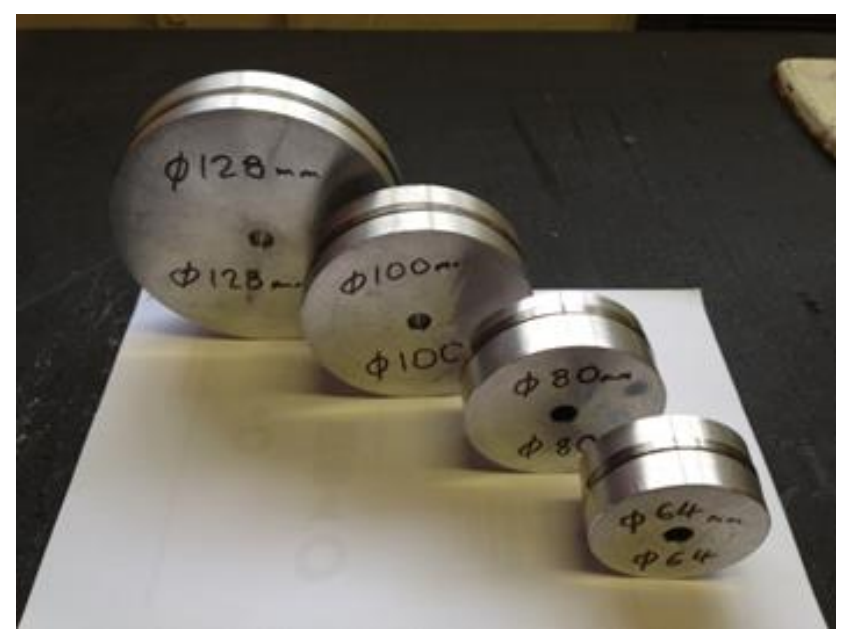

Fig. 5 showing typical roundness formers used 


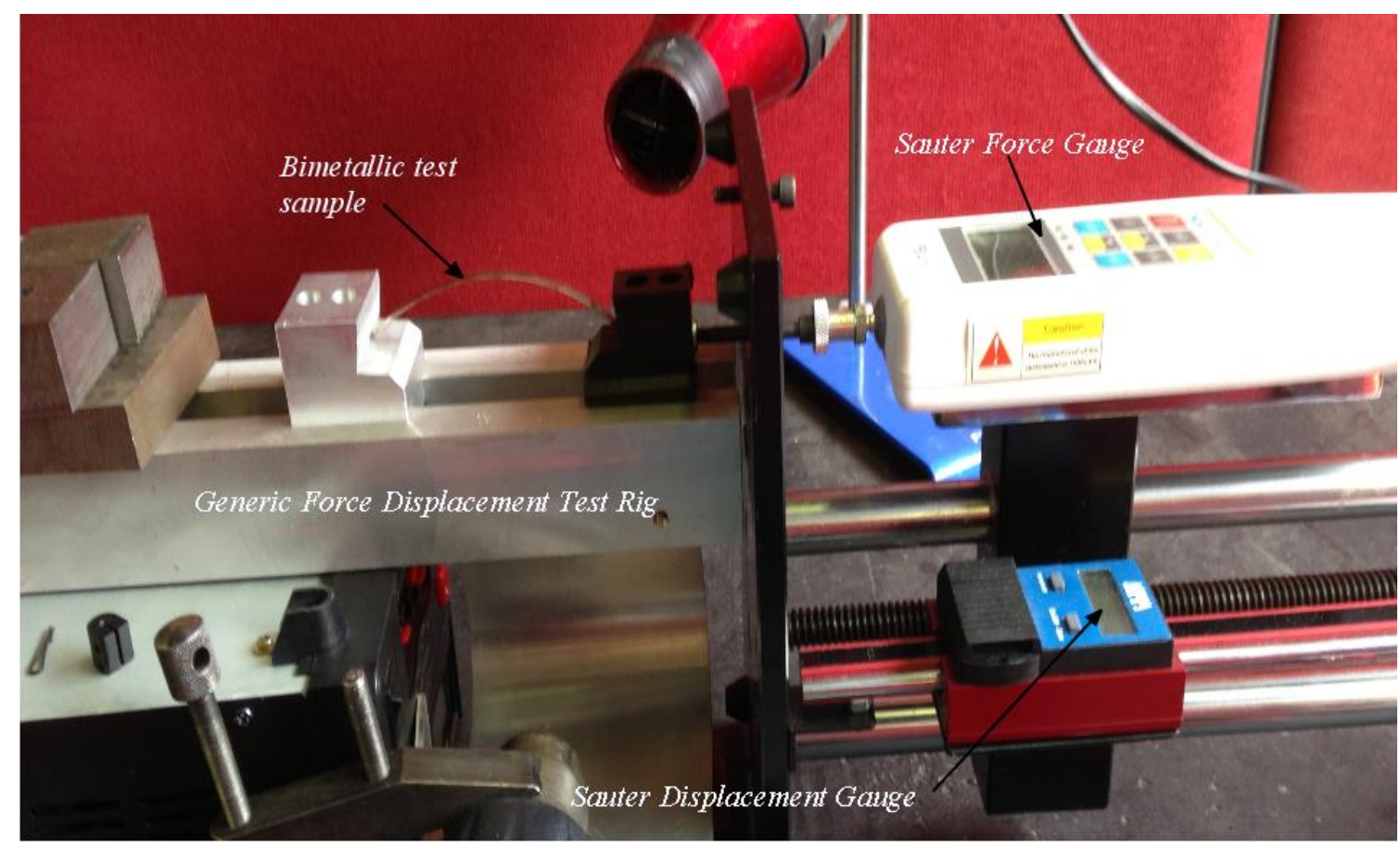

Fig. 7 Generic Force Displacement Test Set up

\subsection{Test Method}

The following test procedure was used for both test sets with all 12 test samples.

a) Test sample mounted into test rig as shown in Fig.7.

b) Force and displacement gauges set to zero.

c) Ambient temperature recorded

d) Test sample displaced by the moving Sauter Force /displacement gauge.

e) Force and displacement values recorded until the maximum displacement achieved.

f) Test sample removed from rig and checked for roundness against former.

g) Steps a) to f) repeated for 3 runs** for all samples in test set.

** Test set 2 only one test run per test sample.

\subsection{Test results}

Both sets of tests yielded excellent force displacement data values which were translated into force displacement curves using Microsoft Excel spread sheet. Due to the simple test setup and the repeatable accuracy of the Sauter force and displacement gauges, very little experimental scatter of data points occurred. Fig. 8 shows the force displacement curves for Test set 1, ie; varying arc lengths $\boldsymbol{A}_{\boldsymbol{b}}$, but keeping the radius of curvature constant at $\boldsymbol{R}_{\boldsymbol{c}}=\mathbf{8 0} \mathbf{m m}$. 


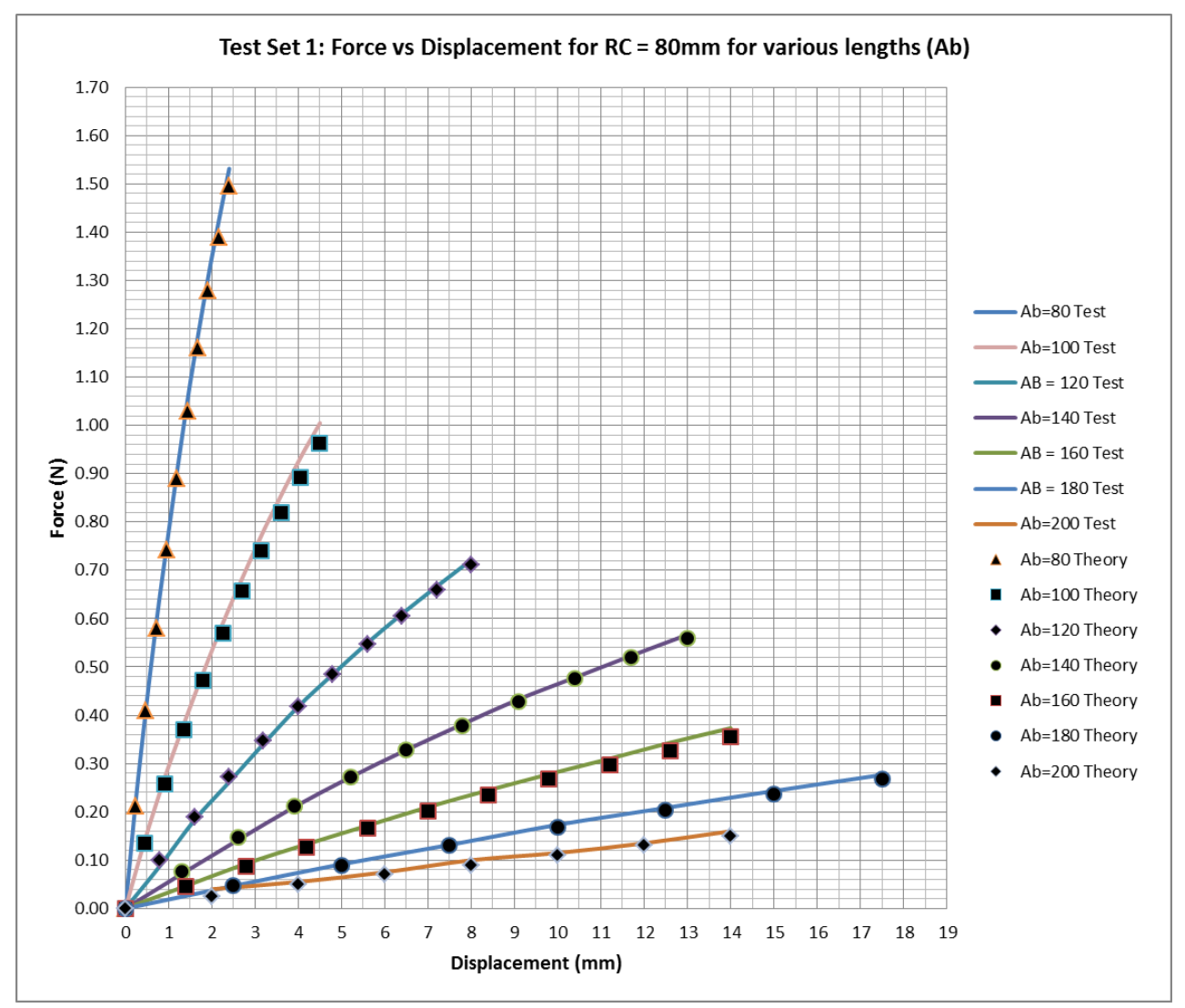

Fig. 8 Test set 1 Comparison of theory to test results

Fig.9 shows the force displacement Test set 2, ie: constant arc length $\boldsymbol{A}_{\boldsymbol{b}}=\mathbf{1 0 0} \mathbf{m m}$, with varying radii of curvature $\boldsymbol{R}_{\boldsymbol{c}}$.

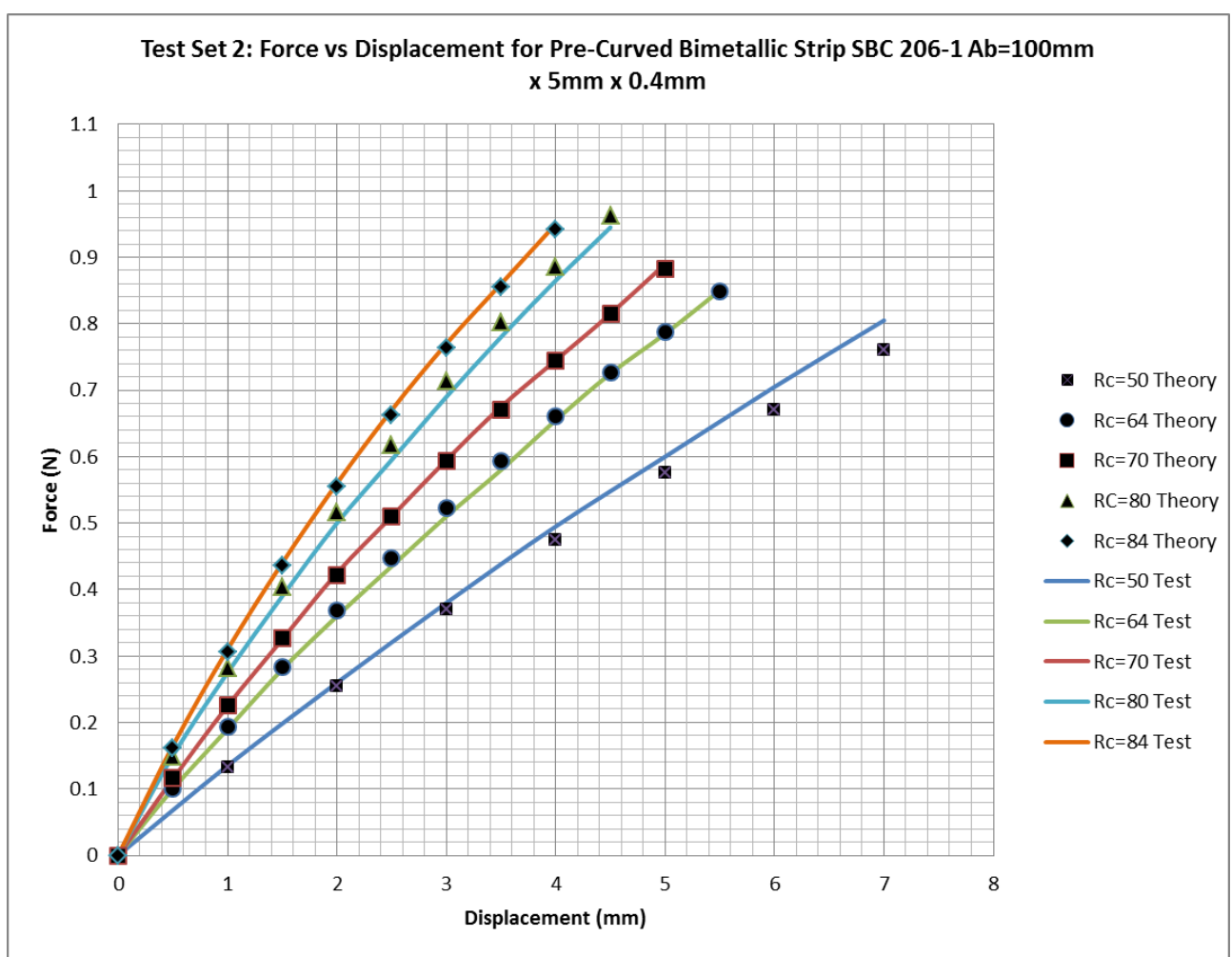

Fig.9 Test set 2 Comparison of theory to test results 


\section{Discussion}

From the test results in the previous section, a good overall correlation exists between the formula introduced here, and the test data from the testing of the 12 sample bimetallic strips. Due to the fact that for test set 2 , only one test run was possible for each test sample, the test data to theory variance was larger in this test set. Comparison must be made with Castigliano's derived force displacement formulae with the same data.

$$
\mathrm{F}_{\text {cast }}:=\frac{2 \cdot \mathrm{E}_{\mathrm{p}} \cdot \mathrm{I} \cdot \mathrm{x}_{\mathrm{d}} \cdot \mathrm{mm}}{\mathrm{R}_{\mathrm{c}}^{3} \cdot\left[\left(\frac{\mathrm{A}_{\mathrm{b}}}{\mathrm{R}_{\mathrm{c}}}\right)-\frac{1}{2} \cdot \sin \left(2 \cdot \frac{\mathrm{A}_{\mathrm{b}}}{\mathrm{R}_{\mathrm{c}}}\right)\right]}
$$

Eqn(35) is the unmodified quadrant derived formula for a semi-circle. This Castigliano derived expression cannot cope with shallow arcs, or large displacements.

$$
F_{c}:=\frac{x_{d} \cdot E_{p} \cdot I}{2 \cdot R_{c}^{3} \cdot\left[\frac{\omega_{c}}{2}+\frac{1}{4}\left(\omega_{c} \cdot \cos \left(\omega_{c}\right)-3 \sin \left(\omega_{c}\right)\right)\right]} \text { eqn.(36) }
$$

Eqn.(36) is Castigliano theorem applied using xy, coordinate geometry in the integration process, however it is limited to a non -varying $\boldsymbol{R}_{\boldsymbol{c}}$, and thus it is a linear expression that does not match well to the test data.

$$
\mathrm{F}_{\mathrm{cd}}:=\frac{\mathrm{x}_{\mathrm{d}} \cdot \mathrm{E}_{\mathrm{p}} \cdot \mathrm{I}}{2 \cdot\left(\mathrm{R}_{\mathrm{d}}\right)^{3} \cdot\left[\frac{\omega_{\mathrm{d}}}{2}+\frac{1}{4}\left(\omega_{\mathrm{d}} \cdot \cos \left(\omega_{\mathrm{d}}\right)-3 \sin \left(\omega_{\mathrm{d}}\right)\right)\right]} \text { eqn.(37) }
$$

Eqn.(37) is the same derivation as eqn.(36), except here, the modified radius of curvature $\boldsymbol{R}_{\boldsymbol{d}}$ replaces $\boldsymbol{R}_{\boldsymbol{c}}$. As can be seen from Fig.10, the application of the variable radius of curvature greatly improves the estimation of the force vs displacement when compared to the fixed radius of curvature $\boldsymbol{R}_{\boldsymbol{c}}$. And lastly, the newly introduced force displacement formula which most closely matches the test data.

$$
\boldsymbol{F}_{\boldsymbol{d}}=\frac{\boldsymbol{M}_{\boldsymbol{d}}-\boldsymbol{M}_{\boldsymbol{c}}}{\frac{\left(\boldsymbol{L}_{\boldsymbol{c}}-x_{d}\right)}{2} \tan \left(\frac{A_{b}}{4 R_{d}}\right)} \text { introduced in section } 2.2
$$

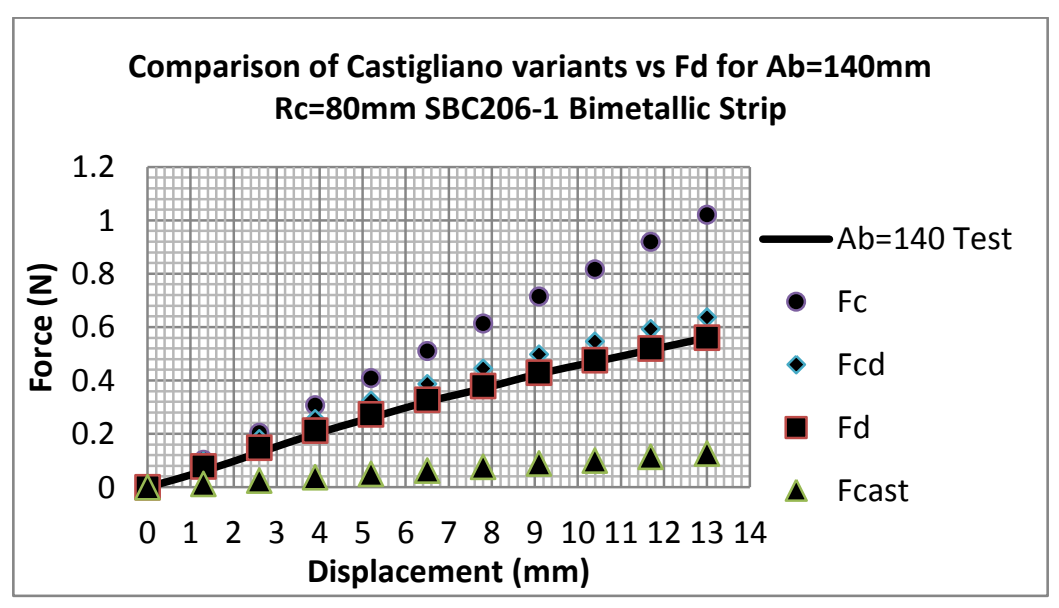

Fig. 10 Comparison of Castigliano to new force displacement formulae $\boldsymbol{F}_{\boldsymbol{d}}$ 


\section{Conclusions}

This work has introduced a new method of calculating the chord line force at a displacement, for a thin, shallow arc, pre-curved bimetallic strip. This work also applies to a single material curved beam that is axially loaded along it's chord line. An important mechanism for evaluating the radius of curvature as a function of chord line displacement was introduced. This new relationship was shown to enhance the accuracy of matching a non-linear force displacement with good results.

It has been shown that the force displacement formula introduced, can deal with relatively large displacements with a reasonably good correlation to the test data.

\section{References}

Abramowitz.M (1972). Handbook of Mathematical Functions Washington D.C., U.S. Government Printing Office.

DAHLBERG.T (2004). Procedure to calculate deflections of curved beams. Int. J. Engng Ed. Great Britain, TEMPUS Publications. 20: 503-513.

Kanthal (2008). Kanthal Thermostatic Bimetal Handbook. Box 502, SE-734 27 Hallstahammar, Sweden, Kanthal.

Shivalik manufacturer of the bimetallic strip http://www.shivalikbimetals.com/home.htm

\section{Appendix}

Table 1

\begin{tabular}{|c|c|c|c|c|c|}
\hline & \multicolumn{3}{|c|}{$A B=80 \mathrm{~mm}$} & \multicolumn{2}{c|}{$R c=80 \mathrm{~mm}$} \\
\hline $\mathbf{X d}$ & $\mathbf{F}(\mathbf{N})$ & $\mathbf{F}(\mathbf{N})$ & $\mathbf{F}(\mathbf{N})$ & Fave & $\mathbf{F d}$ \\
\hline 0.00 & 0.00 & 0.00 & 0.00 & 0.00 & 0.00 \\
\hline 0.24 & 0.22 & 0.22 & 0.23 & 0.22 & 0.21 \\
\hline 0.48 & 0.42 & 0.41 & 0.43 & 0.42 & 0.41 \\
\hline 0.72 & 0.59 & 0.59 & 0.61 & 0.60 & 0.58 \\
\hline 0.96 & 0.75 & 0.75 & 0.77 & 0.76 & 0.74 \\
\hline 1.20 & 0.91 & 0.90 & 0.92 & 0.91 & 0.89 \\
\hline 1.44 & 1.05 & 1.05 & 1.07 & 1.06 & 1.03 \\
\hline 1.68 & 1.18 & 1.18 & 1.20 & 1.19 & 1.16 \\
\hline 1.92 & 1.31 & 1.31 & 1.32 & 1.31 & 1.28 \\
\hline 2.16 & 1.42 & 1.42 & 1.44 & 1.42 & 1.39 \\
\hline 2.40 & 1.53 & 1.53 & 1.54 & 1.53 & 1.50 \\
\hline
\end{tabular}

Table 2

\begin{tabular}{|c|c|c|c|c|c|}
\hline \multicolumn{7}{|c|}{$A B=100 \mathrm{~mm} \quad \mathrm{Rc}=80 \mathrm{~mm}$} & \\
\hline $\mathrm{Xd}$ & $F(N)$ & $F(N)$ & $F(N)$ & $F a v e$ & $F d$ \\
\hline 0.00 & 0.00 & 0.00 & 0.00 & 0.00 & 0.00 \\
\hline 0.45 & 0.15 & 0.15 & 0.14 & 0.14 & 0.13 \\
\hline 0.90 & 0.27 & 0.27 & 0.27 & 0.27 & 0.26 \\
\hline 1.35 & 0.39 & 0.39 & 0.38 & 0.38 & 0.37 \\
\hline 1.80 & 0.49 & 0.49 & 0.49 & 0.49 & 0.47 \\
\hline 2.25 & 0.60 & 0.59 & 0.59 & 0.59 & 0.57 \\
\hline 2.70 & 0.69 & 0.69 & 0.68 & 0.68 & 0.66 \\
\hline 3.15 & 0.78 & 0.78 & 0.77 & 0.77 & 0.74 \\
\hline 3.60 & 0.86 & 0.86 & 0.86 & 0.86 & 0.82 \\
\hline 4.05 & 0.94 & 0.94 & 0.93 & 0.93 & 0.89 \\
\hline 4.50 & 1.01 & 1.01 & 1.01 & 1.01 & 0.96 \\
\hline
\end{tabular}

Table 3

\begin{tabular}{|c|c|c|c|c|c|}
\hline \multicolumn{7}{|c|}{$A B=120 \mathrm{~mm}$} & $R c=80 \mathrm{~mm}$ & \\
\hline $\mathbf{X d}$ & $\mathbf{F}(\mathbf{N})$ & $\mathbf{F}(\mathbf{N})$ & $\mathbf{F}(\mathbf{N})$ & Fave & $\mathbf{F d}(\mathbf{N})$ \\
\hline 0.00 & 0.00 & 0.00 & 0.00 & 0.00 & 0.00 \\
\hline 0.80 & 0.06 & 0.10 & 0.11 & 0.09 & 0.10 \\
\hline 1.60 & 0.19 & 0.17 & 0.20 & 0.18 & 0.19 \\
\hline 2.40 & 0.24 & 0.26 & 0.29 & 0.26 & 0.27 \\
\hline 3.20 & 0.32 & 0.34 & 0.37 & 0.34 & 0.35 \\
\hline 4.00 & 0.40 & 0.42 & 0.44 & 0.42 & 0.42 \\
\hline 4.80 & 0.47 & 0.48 & 0.51 & 0.49 & 0.48 \\
\hline 5.60 & 0.54 & 0.55 & 0.57 & 0.55 & 0.55 \\
\hline 6.40 & 0.60 & 0.61 & 0.63 & 0.61 & 0.61 \\
\hline 7.20 & 0.65 & 0.67 & 0.69 & 0.67 & 0.66 \\
\hline 8.00 & 0.71 & 0.72 & 0.74 & 0.72 & 0.71 \\
\hline
\end{tabular}


Table 3

\begin{tabular}{|c|c|c|c|c|c|}
\hline \multicolumn{5}{|c|}{$A B=140 \mathrm{~mm} \quad R C=80 \mathrm{~mm}$} \\
\hline $\mathbf{X d}$ & $\mathbf{F}(\mathbf{N})$ & $\mathbf{F}(\mathbf{N})$ & $\mathbf{F}(\mathbf{N})$ & Fave & $\mathbf{F d}(\mathbf{N})$ \\
\hline 0.00 & 0.00 & 0.00 & 0.00 & 0.00 & 0.00 \\
\hline 1.30 & 0.06 & 0.08 & 0.08 & 0.07 & 0.08 \\
\hline 2.60 & 0.13 & 0.15 & 0.15 & 0.14 & 0.15 \\
\hline 3.90 & 0.20 & 0.22 & 0.22 & 0.21 & 0.21 \\
\hline 5.20 & 0.26 & 0.28 & 0.28 & 0.27 & 0.27 \\
\hline 6.50 & 0.32 & 0.33 & 0.34 & 0.33 & 0.33 \\
\hline 7.80 & 0.37 & 0.39 & 0.39 & 0.38 & 0.38 \\
\hline 9.10 & 0.43 & 0.44 & 0.44 & 0.43 & 0.43 \\
\hline 10.40 & 0.47 & 0.48 & 0.49 & 0.48 & 0.48 \\
\hline 11.70 & 0.52 & 0.53 & 0.53 & 0.52 & 0.52 \\
\hline 13.00 & 0.56 & 0.57 & 0.57 & 0.57 & 0.56 \\
\hline
\end{tabular}

Table 6

Table 4 Test Set 2
Table 4

Table 5

\begin{tabular}{|c|c|c|c|c|c|}
\hline \multicolumn{7}{|c|}{$A B=160 \mathrm{~mm} \quad \mathrm{RC}=80 \mathrm{~mm}$} \\
\hline $\mathbf{X d}$ & $\mathbf{F}(\mathbf{N})$ & $\mathbf{F}(\mathbf{N})$ & $\mathbf{F}(\mathbf{N})$ & Fave & $\mathbf{F d}(\mathbf{N})$ \\
\hline 0 & 0 & 0 & 0 & 0 & 0 \\
\hline 1.40 & 0.04 & 0.05 & 0.05 & 0.05 & 0.05 \\
\hline 2.80 & 0.09 & 0.10 & 0.10 & 0.09 & 0.09 \\
\hline 4.20 & 0.13 & 0.14 & 0.14 & 0.13 & 0.13 \\
\hline 5.60 & 0.17 & 0.18 & 0.18 & 0.17 & 0.17 \\
\hline 7.00 & 0.21 & 0.21 & 0.22 & 0.21 & 0.20 \\
\hline 8.40 & 0.24 & 0.25 & 0.25 & 0.25 & 0.23 \\
\hline 9.80 & 0.27 & 0.28 & 0.29 & 0.28 & 0.27 \\
\hline 11.20 & 0.31 & 0.31 & 0.32 & 0.31 & 0.30 \\
\hline 12.60 & 0.34 & 0.35 & 0.35 & 0.34 & 0.33 \\
\hline 14.00 & 0.37 & 0.38 & 0.38 & 0.37 & 0.36 \\
\hline
\end{tabular}

Table 7 Table 8

Table 9

Table 6

\section{Test Set 2 Test Set $2 \quad$ Test Set 2 Test Set 2 \\ Test Set 2 Test Set $2 \quad$ Test Set 2 Test Set 2}

\begin{tabular}{|c|c|c|c|c|c|}
\hline \multicolumn{7}{|c|}{$A B=180 \mathrm{~mm} \quad \mathrm{RC}=80 \mathrm{~mm}$} \\
\hline $\mathbf{X d}$ & $\mathbf{F}(\mathbf{N})$ & $\mathbf{F}(\mathbf{N})$ & $\mathbf{F}(\mathbf{N})$ & Fave & $\mathbf{F d}(\mathbf{N})$ \\
\hline 0.00 & 0.00 & 0.00 & 0.00 & 0.00 & 0.00 \\
\hline 2.50 & 0.05 & 0.05 & 0.05 & 0.05 & 0.05 \\
\hline 5.00 & 0.09 & 0.09 & 0.10 & 0.09 & 0.09 \\
\hline 7.50 & 0.13 & 0.13 & 0.14 & 0.13 & 0.13 \\
\hline 10.00 & 0.17 & 0.17 & 0.18 & 0.17 & 0.17 \\
\hline 12.50 & 0.21 & 0.21 & 0.21 & 0.21 & 0.20 \\
\hline 15.00 & 0.24 & 0.24 & 0.25 & 0.24 & 0.24 \\
\hline 17.50 & 0.28 & 0.28 & 0.28 & 0.28 & 0.27 \\
\hline 20.00 & 0.31 & 0.31 & 0.32 & 0.31 & 0.30 \\
\hline 22.50 & 0.34 & 0.34 & 0.35 & 0.34 & 0.33 \\
\hline 25.00 & 0.37 & 0.37 & 0.38 & 0.37 & 0.36 \\
\hline
\end{tabular}

\begin{tabular}{|c|c|c|}
\hline$A b=200$ & \multicolumn{2}{|c|}{$R c=80$} \\
\hline $\mathbf{X d}$ & $\mathbf{F}(\mathbf{N})$ & $\mathbf{F d}(\mathbf{N})$ \\
\hline 0.0 & 0.00 & 0.00 \\
\hline $\mathbf{2 . 0}$ & 0.04 & 0.02 \\
\hline 4.0 & 0.06 & 0.05 \\
\hline 6.0 & 0.08 & 0.07 \\
\hline 8.0 & 0.10 & 0.09 \\
\hline 10.0 & 0.12 & 0.11 \\
\hline 12.0 & 0.14 & 0.13 \\
\hline 14.0 & 0.16 & 0.15 \\
\hline
\end{tabular}

\begin{tabular}{|c|c|c|}
\hline \multicolumn{3}{|c|}{$A b=100, R c=50$} \\
\hline$X d$ & $F(N)$ & $F d$ \\
\hline 0.00 & 0.00 & 0.00 \\
\hline 1.00 & 0.14 & 0.13 \\
\hline 2.00 & 0.26 & 0.25 \\
\hline 3.00 & 0.38 & 0.37 \\
\hline 4.00 & 0.50 & 0.48 \\
\hline 5.00 & 0.60 & 0.58 \\
\hline 6.00 & 0.71 & 0.67 \\
\hline 7.00 & 0.81 & 0.76 \\
\hline 7.50 & 0.85 & 0.81 \\
\hline
\end{tabular}

\begin{tabular}{|c|c|c|}
\hline \multicolumn{3}{|c|}{$A b=100, R c=64$} \\
\hline $\mathbf{X d}$ & $\mathbf{F}(\mathbf{N})$ & $\mathbf{F d}$ \\
\hline 0.00 & 0.00 & 0.00 \\
\hline 0.50 & 0.10 & 0.10 \\
\hline 1.00 & 0.19 & 0.19 \\
\hline 1.50 & 0.28 & 0.28 \\
\hline 2.00 & 0.36 & 0.37 \\
\hline 2.50 & 0.44 & 0.45 \\
\hline 3.00 & 0.51 & 0.52 \\
\hline 3.50 & 0.58 & 0.59 \\
\hline 4.00 & 0.66 & 0.66 \\
\hline 4.50 & 0.73 & 0.73 \\
\hline 5.00 & 0.79 & 0.79 \\
\hline 5.50 & 0.85 & 0.85 \\
\hline
\end{tabular}

\begin{tabular}{|c|c|c|}
\hline \multicolumn{3}{|c|}{$A b=100, R c=70$} \\
\hline$X \mathbf{d}$ & $\mathbf{F}(\mathbf{N})$ & $\mathbf{F d}$ \\
\hline 0 & 0 & 0 \\
\hline 0.50 & 0.12 & 0.12 \\
\hline 1.00 & 0.23 & 0.23 \\
\hline 1.50 & 0.33 & 0.33 \\
\hline 2.00 & 0.43 & 0.42 \\
\hline 2.50 & 0.51 & 0.51 \\
\hline 3.00 & 0.60 & 0.59 \\
\hline 3.50 & 0.68 & 0.67 \\
\hline 4.00 & 0.75 & 0.75 \\
\hline 4.50 & 0.82 & 0.82 \\
\hline 5.00 & 0.89 & 0.88 \\
\hline
\end{tabular}

\begin{tabular}{|c|c|c|}
\hline \multicolumn{3}{|c|}{$A b=100, R c=80$} \\
\hline $\mathbf{X d}$ & $\mathbf{F}(\mathbf{N})$ & $\mathbf{F d}$ \\
\hline 0.00 & 0.00 & 0.00 \\
\hline 0.50 & 0.15 & 0.15 \\
\hline 1.00 & 0.28 & 0.28 \\
\hline 1.50 & 0.39 & 0.40 \\
\hline 2.00 & 0.50 & 0.52 \\
\hline 2.50 & 0.60 & 0.62 \\
\hline 3.00 & 0.69 & 0.71 \\
\hline 3.50 & 0.78 & 0.80 \\
\hline 4.00 & 0.87 & 0.89 \\
\hline 4.50 & 0.95 & 0.96 \\
\hline
\end{tabular}

\begin{tabular}{|c|c|c|}
\hline \multicolumn{3}{|c|}{$A b=100, R c=84$} \\
\hline $\mathbf{X d}$ & $\mathbf{F}(\mathbf{N})$ & $\mathbf{F d}$ \\
\hline 0.00 & 0.00 & 0.00 \\
\hline 0.50 & 0.17 & 0.16 \\
\hline 1.00 & 0.31 & 0.31 \\
\hline 1.50 & 0.44 & 0.44 \\
\hline 2.00 & 0.56 & 0.56 \\
\hline 2.50 & 0.67 & 0.66 \\
\hline 3.00 & 0.77 & 0.76 \\
\hline 3.50 & 0.86 & 0.86 \\
\hline 4.00 & 0.95 & 0.94 \\
\hline
\end{tabular}

Table 10 Comparative data of different Force Displacement formulae

\begin{tabular}{|c|c|c|c|c|c|}
\hline $\mathbf{R c}=\mathbf{8 0}$ & \multicolumn{3}{|c|}{$\mathbf{A b}=\mathbf{1 4 0}$} & \multicolumn{2}{c|}{$\mathbf{E = 2 1 1 G P a}$} \\
\hline $\mathbf{X d}$ & $\mathbf{F}(\mathbf{N})$ & $\mathbf{F d}(\mathbf{N})$ & Fcast & $\mathbf{F c}$ & $\mathbf{F c d}$ \\
\hline 0.000 & 0.000 & 0.000 & 0.000 & 0.000 & 0.000 \\
\hline 1.300 & 0.060 & 0.077 & 0.013 & 0.102 & 0.096 \\
\hline 2.600 & 0.130 & 0.147 & 0.025 & 0.204 & 0.180 \\
\hline 3.900 & 0.200 & 0.212 & 0.038 & 0.306 & 0.256 \\
\hline 5.200 & 0.260 & 0.272 & 0.050 & 0.408 & 0.324 \\
\hline 6.500 & 0.320 & 0.328 & 0.063 & 0.510 & 0.387 \\
\hline 7.800 & 0.370 & 0.379 & 0.075 & 0.613 & 0.444 \\
\hline 9.100 & 0.425 & 0.429 & 0.088 & 0.715 & 0.497 \\
\hline 10.400 & 0.470 & 0.475 & 0.100 & 0.817 & 0.547 \\
\hline 11.700 & 0.515 & 0.520 & 0.113 & 0.919 & 0.593 \\
\hline 13.000 & 0.560 & 0.560 & 0.125 & 1.021 & 0.636 \\
\hline
\end{tabular}

